\title{
Desarrollo de la competencia léxica en el idioma inglés aplicando la estrategia chunking
}

Development of lexical competence in the English language by applying the chunking strategy

Rocío de los Ángeles Barragán Murilloㄹ, Zoila Victoria Herrera Andrade ${ }^{2}$, Esthela Isabel Colcha Guashpa ${ }^{3}$, Daniela Fernanda Guano ${ }^{4}$, Nancy Georgina Rodríguez Arellano ${ }^{5}$

\begin{abstract}
DOI: https://doi.org/10.33262/cienciadigital.v3i2.6.569

The aim of this work is to apply the Chunking Flashcard strategy to develop the lexical competence at Escuela Superior Politécnica de Chimborazo (ESPOCH) to determine its impact on the development of knowledge among second level students. This study revealed how important this study is for the students' learning. This is a quasiexperimental research. The explanatory methodology and the qualitative and quantitative approach were used. The population for this study was second level students of the language center of ESPOCH. It was segmented into the following groups: a control group, a treatment group, and a validation group. A total of 53 students were divided into two groups. Pre-test and post-test were used for the evaluation. A questionnaire was used to gather information. It was used and applied at the beginning and at the end of the study for both the control and treatment groups. Cronbach's alpha was used to validate these instruments. It allowed us to measure the level of reliability and validity. The frequency tables determined the level of dispersion. Decisions were made based on the differences of measures among groups. It is concluded that the use of chunking flashcard strategy helps students improve the lexical competence of the English language.
\end{abstract}

Keywords: Lexical competence, Teacher's training, English language, Cognitive strategy, Chunking strategy.

\section{Resumen}

La presente investigación tiene como objetivo aplicar la estrategia Chunking Flashcards en el desarrollo de la competencia léxica, en la Universidad Escuela Superior Politécnica de Chimborazo, a fin de evidenciar la incidencia que tiene en el desarrollo

\footnotetext{
${ }^{1}$ Escuela Superior Politécnica de Chimborazo, Chimborazo, Ecuador, robarragan@espoch.edu.ec

${ }^{2}$ Escuela Superior Politécnica de Chimborazo, Chimborazo, Ecuador. zoila.herrera@espoch.edu.ec

${ }^{3}$ Escuela Superior Politécnica de Chimborazo, Chimborazo, Ecuador, e_colcha@espoch.edu.ec

${ }^{4}$ Escuela Superior Politécnica de Chimborazo, Chimborazo, Ecuador, daniela.guano@espoch.edu.ec

${ }^{5}$ Escuela Superior Politécnica de Chimborazo, Chimborazo, Ecuador, nancy.rodriguez@espoch.edu.ec
} 
del conocimiento en los estudiantes que cursan el segundo nivel. Revelando la importancia que este estudio aporta a los estudiantes en el aprendizaje. El diseño del estudio es cuasi experimental, La metodología empleada para este caso es de tipo explicativo, con un enfoque cuali-cualitativo y cuantitativo. La población objeto de estudio fue el segundo nivel del centro de idiomas de la ESPOCH segmentado en grupo de control, grupo experimental y grupo de validación, trabajando para la investigación con un total de 53 estudiantes divididos en dos grupos correctamente establecidos, para la evaluación se aplicó el modelo Pre-test-Post-test, formulando un cuestionario para el levantamiento de información, este instrumento fue utilizado y aplicado como prueba de conocimiento al inicio y al final de la intervención tanto al grupo de control como al experimental. Para la validación de estos instrumentos se empleó el coeficiente del Alfa de Cronbach; éste permitió medir su nivel de confianza y fiabilidad. Las tablas de frecuencia determinaron el grado de dispersión. La toma de decisiones se realizó en base a la diferencia de medias existentes en los grupos. Se concluye, que el uso de la estrategia Chunking Flashcards incide positivamente en el desarrollo de la competencia léxica del Idioma Inglés.

Palabras Claves: Competencia Léxica - Capacitación Docente - Idioma Inglés Estrategia Cognitiva - Estrategia Chunking

\section{Introducción}

El Idioma Inglés hoy en día la mayoría de países del mundo se ha convertido en un idioma universal para el ser humano, considerada herramienta fundamental primeramente para incentivar el cerebro, prevenir la demencia, fomentar la memoria y concentración, etc. además abre las puertas y fronteras y se obtiene las posibilidades de mejores oportunidades en cuanto al estudio y trabajo. Considerado el idioma de la comunicación internacional, del comercio y las finanzas.

El inglés es una lengua franca en muchos rincones del planeta y el idioma oficial de muchas Organizaciones Internacionales como la Unión Europea, Naciones Unidas o la Unesco. Esto se debe a razones históricas y políticas, debido a la importancia que tuvo el Imperio Británico en el pasado, cuando extendió su lengua por todos los continentes, y a la importancia como potencia actual de Estados Unidos (Sprachcaffe, 2017)

Los autores García, Clemente y Pérez (1994), exponen: El rol que el docente asume es de gran responsabilidad para mejorar en el proceso de enseñanza aprendizaje del idioma Inglés y cumplir las expectativas que se propongan, para lo cual los docentes deben empezar a desarrollar con sus alumnos la competencia léxica en inglés, ayudando a recordar su léxico buscando a través de nuevas estrategias que le impacte al estudiante, ya sea por partes o subpartes respecto al vocabulario o léxico.

Este trabajo de investigación se efectúa en base a la necesidad de profundizar sobre la temática referente al desarrollo de la competencia léxica de los estudiantes del Centro de Idiomas que cursan el segundo nivel de inglés como segunda lengua extranjera. 
En consecuencia, esta investigación expone la importancia para que el docente sienta la necesidad de investigar sobre esta habilidad, a la vez novedosa y nueva en cuanto a la estrategia que se ha seleccionado llamada Chunking que permite recordar con más facilidad y rapidez su aprendizaje relacionándolo con las Flashcards, las mismas que tienen un poder efectivo en el aprendizaje; muchos de los docentes no conocen muy a fondo, sin embargo lo han usado en forma muy general siendo los estudiantes los principales beneficiarios en esta institución.

Se emplea una metodología explicativa, con un diseño cuali cuantitativo, utilizando el pre y el post test como método de prueba dirigido a estudiantes dividido en dos grupos, obteniendo como resultado a través de la hipótesis planteada en la investigación que al aplicar la estrategia del Chunking incide positiva en el desarrollo de la competencia léxica del Idioma Inglés.

Se puede concluir que el emplear una innovada estrategia para el aprendizaje de los estudiantes como es el Chunking, se constituye en herramientas necesarias para enlazar con los contenidos existentes o generar nuevos que beneficien el aprendizaje eficaz.

\section{Competencia léxica}

En el aprendizaje de cualquier lengua, el desarrollo de la competencia léxica se considera siempre como uno de los pilares fundamentales para el progreso de la competencia comunicativa en ese segundo idioma (De Miguel García, 2004). La ventaja que supone poseer un buen vocabulario que resuelva las necesidades comunicativas es incuestionable; asimismo, la competencia léxica permite expresarnos y comprender lo que se nos manifiesta en la interacción comunicativa. En síntesis, aprender el léxico de una lengua extranjera puede constituir un proceso gratificante si estamos atentos a las palabras y expresiones nuevas, $y$, como en nuestra lengua materna, sentimos curiosidad por descubrir la información que encierran

Para alcanzar mayor comprensión de lo que significa la competencia léxica como subcompetencia dentro de la competencia lingüística y por ende de la competencia comunicativa Orwig (1999) indica que se pueden desarrollar cuatro saberes: la fonología y ortografía, la gramática, el discurso y el vocabulario. Éste último saber es el conocimiento para desarrollar la competencia léxica, la cual a su vez la define como la habilidad para reconocer y usar las palabras de una lengua del mismo modo que los hablantes nativos lo hacen.

\section{Metodología aplicada en la enseñanza del léxico}

Es evidente que la metodología aplicada en la enseñanza del léxico debe basarse en los principios lingüísticos, cognitivos y psicológicos que fundamentan la competencia léxica. Coincidimos con varios autores como Laufer, Marconi, Tréville y Gómez Molina; en que las unidades léxicas son signos lingüísticos que representan fenómenos gramaticales, referenciales, discursivos, socioculturales y estratégicos; de ahí que actúan como instrumentos de desarrollo de la competencia comunicativa. La planificación léxica que el profesor desarrolla en el aula debe tomar en consideración dicha propuesta epistemológica y, por ello, 
debe seleccionar el tipo de unidades léxicas más adecuadas para desarrollar cada uno de los subcomponentes integrantes de la competencia léxica: palabras, colocaciones, locuciones, paremias, rutinas, etc. De Miguel García (2004: 02).

\section{Dimensiones de la competencia léxica}

Es evidente que la metodología aplicada a la enseñanza del léxico debe basarse en los principios linguiísticos, cognitivos y psicológicos que fundamentan la competencia léxica. Los distintos estudios que se centran en la definición, descripción y enumeración de las dimensiones de la competencia léxica, se pueden comprender mejor si las agrupamos en torno a varios ejes:

- La dimensión lingüística, manifestada a través del análisis de la complejidad del significado y de la estructura de las palabras que se basa en una descripción de los distintos aspectos que implica conocer una palabra a partir de su aspecto fonológico, morfológico, sintáctico y semántico.

- La dimensión sociolingüística, que implica el conocimiento de las distintas dimensiones lingüísticas y el uso de los determinados aspectos de una palabra en contextos determinados.

- La dimensión psicolingüística representada en los supuestos del reconocimiento de la palabra, en el conocimiento receptivo y productivo, además en la distinción entre conocimiento declarativo y procedimental que, con ayuda de la dimensión pedagógica se refleja en el análisis tanto de la dificultad de adquirir la competencia léxica como de los distintos tipos de conocimiento y las destrezas activadas en el aprendizaje. (ELE, 2007)

\section{Formación de palabras}

La terminología formación o configuración de palabras, hace referencia a las diferentes técnicas que existen para componer palabras. La licenciada Castelli (2016) describe con detalle las lenguas cuentan con distintos procedimientos para formar palabras nuevas. Dependiendo del procedimiento empleado las palabras en formación pueden dividirse en: derivadas, compuestas o palabras parasintéticas.

Palabras: El autor Apostilla (2014) define "la palabra es un conjunto o secuencia de sonidos articulados, que se pueden representar gráficamente con letras, y por lo general, asocian un significado". En efecto, el concepto de palabra es un conjunto de letras y sonidos para luego tener un significado.

Raíces: La raíz de una palabra, también llamada lexema está vinculada directamente a la esencia de su significado, es esa parte que no cambia y que, si se escribe con diferentes terminaciones, da origen a varias palabras de una misma familia. Según la publicación del autor Pablo (2007) en las palabras existe un monema que no puede faltar, puesto que es el que le da sentido, el que porta el significado original.

Conjuntos de Palabras: Un concepto de conjunto de palabras, viene dado por la palabra conjunto que es un grupo de elemento dentro de un espacio definido; de la misma forma un conjunto de palabras hace alusión a un grupo de términos de la misma familia, de los que se puede disponer de manera coherente. Para ello, es necesario entender, además, la definición de 
familia de palabras y según una publicación de (WoodwarSpanish, 2017) es el grupo de palabras que derivan de una misma palabra primitiva, tienen una raíz en común y cierta relación en su significado.

\section{Partes del Habla}

Se puede definir a las partes del habla como las palabras básicas que tiene un idioma, es necesario entender, además, que no todos los idiomas tienen las mismas partes del habla, por lo tanto, no pueden ser generalizadas. Se explica que, en este caso, se menciona las partes del habla en el idioma inglés y para ello se citará al docente José Colmenares (2015), quién explica que las partes del habla de este idioma son: el nombre, el verbo, los adjetivos, los adverbios, el pronombre, la conjunción, la preposición, la interjección, el artículo y el numeral.

\section{Contenidos Temáticos}

La terminología "Contenidos temáticos" hace alusión a los diferentes temas que se incluyen en un trabajo de investigación o en cualquier tipo de documento que contenga más de un tópico, generalmente se utilizan para dar orden a las descripciones narrativas de los temas que se van a tratar a lo largo del escrito. (Pérez, J., \& Gardney, A. , 2009)

\section{Idioma Inglés}

Al hablar del idioma inglés de acuerdo al fenómeno de la globalización (Rodrìguez, 2014) expresa que se convierte en uno de los activos más importantes del conocimiento dentro de la esfera social por cuanto en la sociedad se han suscitado nuevos campos laborales en el orden tecnológico, comercial, político, académico y cultural donde el idioma inglés abarca significativamente nuevos espacios creando un liderazgo imprescindible en el mercado nacional e internacional.

\section{El inglés como lengua mundial}

Como indica Fernández (1964), durante el siglo XVI, en pleno colonialismo Europeo, el Francés y el Italiano eran las lenguas que permitían la comunicación global, pero solo entre las elites mientras que el Holandés y el Español eran las lenguas esenciales para aquellos que viajaran a América, África y Asia, sin embargo a partir del siglo XVIII el Idioma Inglés fue perfilándose como una potencial lengua global a través de victorias militares, el proceso de industrialización y las reformas llevadas a cabo al interior de Gran Bretaña (Wright 2004) y durante el siglo XIX esta situación se consolidó gracias en gran medida a la expansión del Imperio Británico, lo cual disipó cualquier duda que pudiese quedar en cuanto a la importancia de la lengua inglesa en el mundo.

\section{El inglés como idioma extranjero}

El idioma inglés se ha convertido en una herramienta considerablemente útil en los últimos años en el mundo laboral y social, debido a que muchas empresas de diversos sectores económicos incluyen como requisito para poder formar parte de ellas tener un cierto grado de dominio de este idioma además de las variadas aplicaciones relacionados con nuestra vida 
social y recreativa hacen del idioma inglés una llave que abre puertas a horizontes que de otra manera permanecerían cerrados. (Ceballos, K., Dàvila, N., Espioza, J. \& Ramìrez, M., 2014).

\section{Importancia del inglés en la actualidad}

(Cabanilla, 2018) Un estudio de la reclutadora Hays Colombia encontró que dominar el inglés aumenta en un $50 \%$ las posibilidades de conseguir un mejor empleo y, en promedio, incrementa en un $35 \%$ el salario de un profesional. Por tanto las oportunidades laborales se multiplicarán en cuanto dominemos el idioma. Tanto en áreas gubernamentales como en empresas multinacionales, sin importar el campo de trabajo, el inglés aportará siempre ventajas a la hora de ascender o acceder a otro puesto de trabajo, mejorando su situación laboral de hoy.

\section{Capacitación Docente}

Entre los autores citaremos a Camargo, Clavo, Franco, Vergara, Londoño, Zapata y Garavito, (2018) quienes exponen que la formación permanente (o capacitación) del docente debe entenderse como un proceso de actualización que le posibilita realizar su práctica pedagógica y profesional de una manera significativa, pertinente y adecuada a los contextos sociales en que se inscribe y a las poblaciones que atiende. Es así como dicha formación se presenta articulada al ejercicio mismo de la práctica pedagógica y a formas de entenderla e inscribirla en los contextos sociales donde se realiza, y; en esta misma forma se concibe al docente como un profesional capacitado (idóneo) para reconstruir el conocimiento pedagógico, a partir de la experiencia a que se enfrenta el día al día.

\section{La Capacitación Docente y la Calidad de la Educación}

La centrabilidad de la educación y el conocimiento para promover el desarrollo de las naciones en el marco de la moderna ciudadanía y de la competitividad internacional (Cepal/Unesco, 199: 125) pone a los maestros en un lugar privilegiado, como ejes de la producción material y cultural de la sociedad. La educación está llamada a responder con calidad al reto de modernizar la sociedad y construir una nación justa y democrática. "Una mayor y mejor contribución del sistema educativo y de la actuación de los educadores a la construcción de la modernidad, la democracia y la apropiación de deberes pertinentes a nuestro modelo de desarrollo, significa un incremento en la calidad de la educación" Toro, (1996: 6)

\section{La relación teoría-práctica}

Plantear la relación entre teoría y práctica asociada a las necesidades de formación de los maestros lleva a considerar por lo menos tres situaciones: la relación con los modelos pedagógicos, el pensamiento del profesor y la relación teoría-práctica en los procesos de formación de los docentes:

a.- Los modelos pedagógicos: Son procesos formativos en su singularidad (didáctica) que relaciona a los mundos de la vida y de la escuela mediante la selección y la sistematización del 
mundo real, concretando en aquella parte de la cultura que se selecciona, para que inmersa en el proceso educativo, posibilite la formación del educando (Gonzàlez, 1996)

b.- El pensamiento del profesor: Es fundamental tener una mirada integral de la práctica para reconocer todos los aspectos que se reflejan en ella, y que es necesario revisar el alcance de las decisiones que se toman desde los modelos pedagógicos (Callejas, 1986).

c.- La relación teoría-práctica en los procesos de formación de los docentes: Los estudios de Schiefelbein y Schiefelbein (1998) mantienen la tendencia a privilegiar la práctica dentro de los procesos de formulación y de actualización de los docentes, en cuanto este conocimiento por la acción afectaría las pedagogías frontales y las prácticas tradicionales de aula

\section{Estrategia}

Existen varios autores que definen el concepto de estrategia relacionada al aprendizaje danto mayor énfasis a los autores (Dansereau, 1986, pág. 315) son las que "definen como secuencias articuladas de procedimientos o actividades que se seleccionan con el objetivo de permitir la consecución, acopio y manejo de la información". Estos autores mantienen la misma idea en común en que son procedimientos y actividades de hacer algo, esas actividades actualmente nos son notorias en los docentes ya que por la ignorancia de aquella no logran el objetivo del aprendizaje.

\section{Estrategias Cognitivas}

Para Oxford (1990), estrategias cognitivas son acciones intelectuales implementadas esencialmente para reflexionar sobre el propio aprendizaje, analizar y sintetizar lo aprendido. Gracias a estas estrategias se transforma lo aprendido, se practican o se ensayan los nuevos conocimientos, se razona deductivamente, se interpretan expresiones, se realizan análisis contrastivos entre los idiomas, se hacen traducciones y transferencias, y ofrecen la posibilidad de crear estructuras.

\section{Estrategias Chunking}

Las flash cards, conocidas en español como tarjetas de aprendizaje, tarjetas educativas, son tarjetas que contienen palabras, imágenes, símbolos o números en uno o ambos lados y se usan para adquirir conocimientos al memorizar su contenido mediante el repaso espaciado del conjunto de tarjetas. Estas tarjetas de estudio estimulan la memoria activa relacionando una pregunta que se nos hace con una respuesta correcta proporcionada por la tarjeta y espaciando cada vez más la misma tarjeta al responder correctamente, ya que se va fijando en la memoria. (Cohn, 2009)

\section{Metodología}

En esta investigación se aplicó un estudio de diseño cuasi experimental, se basó en la técnica de pruebas, diseñando un pre test previo a su aplicación a fin de analizar el equivalente entre los grupos; puesto que se trabaja con registros de control, y se aplica a quienes se los denomina 
grupo experimental y de control con el propósito de verificar las variables luego de la aplicación del post test.

Este trabajo de investigación tiene un enfoque mixto (cuali-cuantitativo); en el enfoque cualitativo describe cualidades que se usan en el análisis de los cuestionarios; mientras que en el enfoque cuantitativo se puede probar la hipótesis en base a la medición numérica y el análisis estadístico, lo que se permite establecer patrones de comportamiento y comprobación de variables,

El nivel de esta investigación es explicativo, su propósito es demostrar que los cambios en la variable dependiente (aprendizaje de destrezas) fueron causados por la variable independiente (recurso didáctico Chunking).

El tipo de la investigación es de campo con carácter descriptivo obteniendo los datos directamente de los alumnos con la ayuda de docentes del Centro de Idiomas de la ESPOCH. "O” que significa que los/las docentes apoyaron en la recolección de datos del grupo control, mientras que los/las estudiantes formaron parte de un grupo experimental y uno de control para tener claro una visión del problema de estudio, a través de los instrumentos pre test y post test.

La Población y muestra a considerarse para la investigación se ubicó en el Centro de Idiomas como Unidad Académica de la ESPOCH, en el periodo académico abril-agosto 2017; constituida por los estudiantes de los cursos "G" "H" y "O" del Segundo Nivel del Centro de Idiomas, integrada por treinta y uno (31), treinta y ocho (38) y veintitrés (23) estudiantes respectivamente dando un total de noventa y dos estudiantes.

Como grupo experimental se trabajó con el paralelo "O" con quienes se aplicó CHUNKING FLASHCARD estrategia didáctica para determinar su influencia en el desarrollo de destrezas en el aprendizaje del inglés. Como grupo de control se trabajó con el paralelo " $\mathrm{H}$ " con quienes se utilizó la metodología cotidiana de empleo de recurso audiovisual y la validación del instrumento lo realizó con el paralelo "G" del Segundo Nivel. Así la población estaba constituida por cincuenta y tres estudiantes (53), de los cuales se ha obtenido la información necesaria directamente de la secretaría del Centro de Idiomas.

Los Métodos de investigación utilizados en este trabajo fueron: Científico, AnalíticoComparativo, Estadístico y Lógico. Así como las técnicas e instrumentos: la entrevista y encuesta, a través de cuestionarios para la recolección de datos y guías de preguntas.

Una vez obtenidos los resultados en primera instancia se usó las herramientas estadísticas para lo cual se realiza el procesamiento de resultados mediante la distribución de frecuencias y representaciones gráficas, empleando las medidas de tendencia central: la media, mediana, y moda y las medidas de dispersión: varianza y desviación estándar, así también para la prueba de hipótesis la prueba estadística t student que se utilizó por tratarse de muestras pequeñas, como programa de computo se empleó la hoja de cálculo Excel 2016. 


\section{Resultados}

Gráfico 1. Pre test grupo de control análisis global de satisfacción

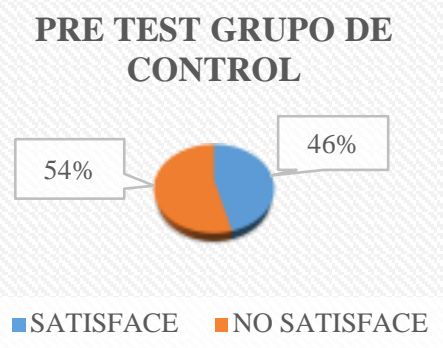

Fuente: Secretaría del Centro de Idiomas ESPOCH

Gráfico 2. Pre test grupo experimental análisis global de satisfacción

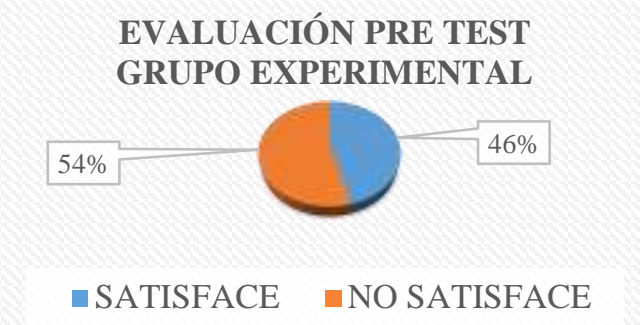

Fuente: Secretaría del Centro de Idiomas ESPOCH

Gráfico 3. Post test grupo de control análisis global de satisfacción

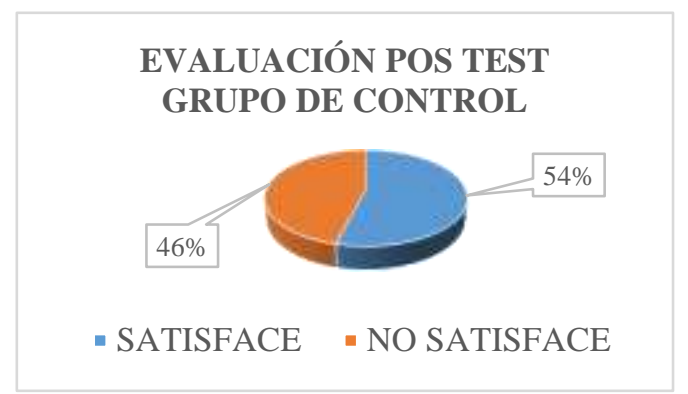

Fuente: Secretaría del Centro de Idiomas ESPOCH

Gráfico 4. Post test grupo experimental análisis global de satisfacción

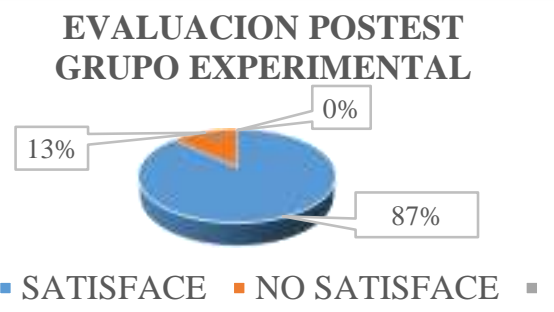

Fuente: Secretaría del Centro de Idiomas ESPOCH 


\section{Analisis de resultados}

Tabla 1. De frecuencias para medidas de tendencia central pre test grupo de control

\begin{tabular}{cccccc}
\hline No. & $\begin{array}{c}\text { Clases o } \\
\text { Grupos } \\
\mathrm{xi}\end{array}$ & $\begin{array}{c}\text { Frecuencia } \\
\text { Absoluta } \\
\mathrm{Fi}\end{array}$ & $\begin{array}{c}\text { Frecuencia } \\
\text { absoluta } \\
\text { acumulada }\end{array}$ & $\begin{array}{c}\text { Frecuencia } \\
\text { relativa }\end{array}$ & $\begin{array}{c}\text { Frecuencia } \\
\text { relativa } \\
\text { acumulada }\end{array}$ \\
\hline 1 & 3 & 5 & 5 & 0,17 & 0,17 \\
2 & 4 & 10 & 15 & 0,33 & 0,50 \\
3 & 5 & 10 & 25 & 0,33 & 0,83 \\
4 & 7 & 4 & 29 & 0,13 & 0,97 \\
5 & 8 & 1 & 30 & 0,03 & 1,00 \\
& TOTAL & 30 & & 1,00 & \\
\hline
\end{tabular}

Fuente: Secretaría del Centro de Idiomas ESPOCH

Media $\bar{x}=\frac{1}{n} \sum_{i=1}^{n} x i=\frac{x_{1}+x_{2}+\ldots+x_{n}}{n}=\frac{\sum x i}{n}=4.7$

Moda $M o=$ Valor que más se repite $=4$

Mediana $M e=(n+1) / 2=5$

Rango $R=X_{\text {máximo }}-X_{\text {mínimo }}=5$

Tabla 2 De frecuencia para medidas de dispersión pre test grupo de control

\begin{tabular}{ccccccc}
\hline No. & $\mathrm{xi}$ & $\mathrm{Fi}$ & $\mathrm{xi}^{\star} \mathrm{fi}$ & $\mathrm{d}$ & $\mathrm{d}^{\wedge} 2$ & $\mathrm{fi}^{*} \mathrm{~d}^{\wedge} 2$ \\
\hline 1 & 3 & 5 & 15 & $-1,7$ & 2,89 & 14,45 \\
2 & 4 & 10 & 40 & $-0,7$ & 0,49 & 4,90 \\
3 & 5 & 10 & 50 & 0,3 & 0,09 & 0,90 \\
4 & 7 & 4 & 28 & 2,3 & 5,29 & 21,16 \\
5 & 8 & 1 & 8 & 3,3 & 10,89 & 10,89 \\
TOTAL & & 30 & 141 & & & 52,30 \\
\hline
\end{tabular}

Fuente: Secretaría del Centro de Idiomas ESPOCH

Varianza $\sigma_{n}^{2}=\sum_{i=1}^{n}(x i-\bar{x})^{2}=\frac{\sum f i * d^{2}}{N}=1.74333 \ldots$

Desviación Estándar $\sigma=\sqrt{\frac{\sum f i * d^{2}}{n}}=1.3203$

Coeficiente de variación $C_{v}=\frac{\sigma}{\bar{X}}=28 \%$

$$
\begin{gathered}
\bar{X}+\sigma ; \bar{X}-\sigma \\
6.0 ; 3.4
\end{gathered}
$$

El mayor número de calificaciones cae dentro del rango que va desde 6.0 puntos hacia arriba hasta 3.4 puntos hacia abajo; puesto que la desviación estándar 1.32 representa el grado de dispersión que existe entre los valores de la variable con la media aritmética. 
Tabla 3 De frecuencias para medidas de tendencia central pre test grupo experimental

\begin{tabular}{cccccc}
\hline No. & $\begin{array}{c}\text { Clases o } \\
\text { Grupos } \\
\text { xi }\end{array}$ & $\begin{array}{c}\text { Frecuencia } \\
\text { absoluta } \\
\mathrm{fi}\end{array}$ & $\begin{array}{c}\text { Frecuencia } \\
\text { absoluta } \\
\text { acumulada }\end{array}$ & $\begin{array}{c}\text { Frecuencia } \\
\text { relativa }\end{array}$ & $\begin{array}{c}\text { Frecuencia } \\
\text { relativa } \\
\text { acumulada }\end{array}$ \\
\hline 1 & 3 & 4 & 4 & 0,17 & 0,17 \\
2 & 4 & 10 & 14 & 0,43 & 0,61 \\
3 & 5 & 4 & 18 & 0,17 & 0,78 \\
4 & 6 & 1 & 19 & 0,04 & 0,83 \\
5 & 7 & 4 & 23 & 0,17 & 1,00 \\
TOTAL & & 23 & 78 & 1,00 & \\
\hline
\end{tabular}

Fuente: Secretaría del Centro de Idiomas ESPOCH

Media $\bar{x}=\frac{1}{n} \sum_{i=1}^{n} x i=\frac{x_{1}+x_{2}+\ldots+x_{n}}{n}=\frac{\sum x i}{n}=4.6086$

Moda $M o=$ Valor que más se repite $=4$

Mediana $\boldsymbol{M e}=(\boldsymbol{n}+\mathbf{1}) / \mathbf{2}=4$

Rango $\boldsymbol{R}=\boldsymbol{X}_{\text {máximo }}-\boldsymbol{X}_{\text {mínimo }}=4$

Tabla 4 De frecuencias para medidas de dispersión pre test grupo experimental

\begin{tabular}{ccccccc}
\hline No. & $\mathrm{xi}$ & $\mathrm{fi}$ & $\mathrm{xi}^{\star} \mathrm{fi}$ & $\mathrm{d}$ & $\mathrm{d}^{\wedge} 2$ & $\mathrm{fi}^{*} \mathrm{~d}^{\wedge} 2$ \\
\hline 1 & 3 & 4 & 12 & $-1,6$ & 2,59 & 10,35 \\
2 & 4 & 10 & 40 & 4,0 & 16,00 & 160,00 \\
3 & 5 & 4 & 20 & 1,0 & 1,00 & 4,00 \\
4 & 6 & 1 & 6 & 2,0 & 4,00 & 4,00 \\
5 & 7 & 4 & 28 & 3,0 & 9,00 & 36,00 \\
TOTAL & & 23 & 25 & & & 214,35 \\
\hline
\end{tabular}

Fuente: Secretaría del Centro de Idiomas ESPOCH

Varianza $\sigma_{n}^{2}=\sum_{i=1}^{n}(x i-\bar{x})^{2}=\frac{\sum f i * d^{2}}{N}=1.74333 \ldots$

Desviación Estándar $\sigma=\sqrt{\frac{\sum f i * d^{2}}{n}}=1.3203$

Coeficiente de variación $C_{v}=\frac{\sigma}{\bar{X}}=28 \%$

$$
\begin{gathered}
\bar{X}+\sigma ; \bar{X}-\sigma \\
7.7 ; 1.6
\end{gathered}
$$

El mayor número de calificaciones cae dentro del rango que va desde 7.7 puntos hacia arriba hasta 1.6 puntos hacia abajo; puesto que la desviación estándar 3.05 representa el grado de dispersión que existe entre los valores de la variable con la media aritmética. 
Tabla 5 De frecuencias para medidas de tendencia central post test grupo de control

\begin{tabular}{rccccc}
\hline No. & $\begin{array}{c}\text { Clases o } \\
\text { Grupos } \\
\mathrm{xi}\end{array}$ & $\begin{array}{c}\text { Frecuencia } \\
\text { absoluta } \\
\mathrm{fi}\end{array}$ & $\begin{array}{c}\text { Frecuencia } \\
\text { absoluta } \\
\text { acumulada }\end{array}$ & $\begin{array}{c}\text { Frecuencia } \\
\text { relativa }\end{array}$ & $\begin{array}{c}\text { Frecuencia } \\
\text { relativa } \\
\text { acumulada }\end{array}$ \\
\hline 1 & 4 & 7 & 7 & 0,23 & 0,23 \\
2 & 5 & 9 & 16 & 0,30 & 0,53 \\
3 & 6 & 8 & 24 & 0,27 & 0,80 \\
4 & 7 & 5 & 29 & 0,17 & 0,97 \\
5 & 8 & 1 & 30 & 0,03 & 1,00 \\
TOTAL & & 30 & & 1,00 & \\
\hline
\end{tabular}

Fuente: Secretaría del Centro de Idiomas ESPOCH

Media $\bar{x}=\frac{1}{n} \sum_{i=1}^{n} x i=\frac{x_{1}+x_{2}+\cdots+x_{n}}{n}=\frac{\sum x_{i}}{n}=5.5$

Moda $=$ Valor que más se repite $=5$

Mediana $M e=(n+1) / 2=5$

Rango $R=x_{\text {máximo }}-x_{\text {mínimo }}=4$

Tabla 6 De frecuencias para medidas de dispersión post test grupo de control

\begin{tabular}{rcccccc}
\hline No. & $\mathrm{xi}$ & $\mathrm{fi}$ & $\mathrm{xi}^{\star} \mathrm{fi}$ & $\mathrm{d}$ & $\mathrm{d}^{\wedge} 2$ & $\mathrm{fi}^{\star} \mathrm{d}^{\wedge} 2$ \\
\hline 1 & 4 & 7 & 28 & $-1,5$ & 2,15 & 15,06 \\
2 & 5 & 9 & 45 & $-0,5$ & 0,22 & 1,96 \\
3 & 6 & 8 & 48 & 0,5 & 0,28 & 2,28 \\
4 & 7 & 5 & 35 & 1,5 & 2,35 & 11,76 \\
5 & 8 & 1 & 8 & 2,5 & 6,42 & 6,42 \\
TOTAL & & 30 & 164 & & & 37,47 \\
\hline
\end{tabular}

Fuente: Secretaría del Centro de Idiomas ESPOCH

Varianza $\sigma_{n}^{2}=\sum_{i=1}^{n}(x i-\bar{x})^{2}=\frac{\sum f i \times d^{2}}{N}=1,2488889$

Desviación Estándar $\sigma=\sqrt{\frac{\sum f i \times d^{2}}{n}}=1,117537$

Coeficiente de Variación $C_{v}=\frac{\sigma}{\bar{x}}=20 \%$

$\bar{x}+\sigma ; \bar{x}-\sigma$

6,$6 ; 4,3$

El mayor número de calificaciones cae dentro del rango que va desde 6.6 puntos hacia arriba hasta 4.3 puntos hacia abajo; puesto que la desviación estándar 1.12 representa el grado de dispersión que existe entre los valores de la variable con la media aritmética. 
Tabla 7 De frecuencias para medidas de tendencia central post test grupo experimental

\begin{tabular}{cccccc}
\hline No. & $\begin{array}{c}\text { Clases o } \\
\text { Grupos } \\
\mathrm{xi}\end{array}$ & $\begin{array}{c}\text { Frecuencia } \\
\text { Absoluta } \\
\mathrm{Fi}\end{array}$ & $\begin{array}{c}\text { Frecuencia } \\
\text { absoluta } \\
\text { acumulada }\end{array}$ & $\begin{array}{c}\text { Frecuencia } \\
\text { relativa }\end{array}$ & $\begin{array}{c}\text { Frecuencia } \\
\text { relativa } \\
\text { acumulada }\end{array}$ \\
\hline 1 & 6 & 1 & 1 & 0,04 & 0,04 \\
2 & 7 & 1 & 2 & 0,04 & 0,09 \\
3 & 8 & 5 & 7 & 0,22 & 0,30 \\
4 & 9 & 12 & 19 & 0,52 & 0,83 \\
5 & 10 & 4 & 23 & 0,17 & 1,00 \\
& TOTAL & 23 & & 1,00 & \\
\hline
\end{tabular}

Fuente: Secretaría del Centro de Idiomas ESPOCH

Media $\bar{x}=\frac{1}{n} \sum_{i=1}^{n} x i=\frac{x_{1}+x_{2}+\cdots+x_{n}}{n}=\frac{\sum x_{i}}{n}=8.7$

Moda $=$ Valor que más se repite $=9$

Mediana $M e=(n+1) / 2=9$

Rango $R=x_{\text {máximo }}-x_{\text {mínimo }}=4$

Tabla 8 De frecuencias para medidas de dispersión post test grupo experimental

\begin{tabular}{ccccccc}
\hline No. & $\mathrm{xi}$ & $\mathrm{fi}$ & $\mathrm{xi}^{\star} \mathrm{fi}$ & $\mathrm{d}$ & $\mathrm{d}^{\wedge} 2$ & $\mathrm{fi}^{\star} \mathrm{d}^{\wedge} 2$ \\
\hline 1 & 6 & 1 & 6 & $-2,7$ & 7,50 & 7,50 \\
2 & 7 & 1 & 7 & $-1,7$ & 3,02 & 3,02 \\
3 & 8 & 5 & 40 & $-0,7$ & 0,55 & 2,73 \\
4 & 9 & 12 & 108 & 0,3 & 0,07 & 0,82 \\
5 & 10 & 4 & 40 & 1,3 & 1,59 & 6,36 \\
& TOTAL & 23 & 201 & & & 20,43 \\
\hline
\end{tabular}

Fuente: Secretaría del Centro de Idiomas ESPOCH

Varianza $\sigma_{n}^{2}=\sum_{i=1}^{n}(x i-\bar{x})^{2}=\frac{\sum f i \times d^{2}}{N}=0,8884688$

Desviación Estándar $\sigma=\sqrt{\frac{\sum f i \times d^{2}}{n}}=0,9425862$

Coeficiente de Variación $C_{v}=\frac{\sigma}{\bar{x}}=11 \%$

$\bar{x}+\sigma ; \bar{x}-\sigma$

9,$7 ; 7,8$

El mayor número de calificaciones cae dentro del rango que va desde 9.7 puntos hacia arriba hasta 7.8 puntos hacia abajo; puesto que la desviación estándar 0.94 representa el grado de dispersión que existe entre los valores de la variable con la media aritmética.

Análisis estadístico del pre test aplicado al grupo de control 


\section{Distribución de frecuencias 01}

\begin{tabular}{cccccc}
\hline$O_{1_{i}}$ & $\mathrm{fi}$ & $\mathrm{hi}$ & $O_{1_{i}} * f i$ & $O_{1_{i}}^{2}$ & $O_{1_{i}}^{2} * f i$ \\
\hline 3 & 5 & 0,17 & 15 & 9 & 45 \\
4 & 10 & 0,33 & 40 & 16 & 160 \\
5 & 10 & 0,33 & 50 & 25 & 250 \\
7 & 4 & 0,13 & 28 & 49 & 196 \\
8 & 1 & 0,03 & 8 & 64 & 64 \\
TOTALES & 30 & 1,00 & 141 & 163 & 715 \\
\hline
\end{tabular}

Fuente: Secretaría del Centro de Idiomas ESPOCH

1. Cálculo de los estadígrafos

- Media Aritmética (Promedio $\mathrm{O}_{1}$ )

$$
\begin{aligned}
& \overline{O_{1}}=\frac{\sum_{i=1}^{n} O_{1_{i}} * f i}{n} \\
& \overline{O_{1}}=\frac{141}{30} \\
& \overline{O_{1}}=4,7
\end{aligned}
$$

- Desviación Estándar

La desviación estándar nos permite conocer el grado de dispersión de los datos en relación a la media aritmética.

$$
\begin{aligned}
S_{O_{1}} & =\sqrt{\frac{\sum f i * O_{1_{i}}^{2}-\frac{\left(\sum f i * O_{1_{i}}\right)^{2}}{n-1}}{n}} \\
S_{O_{1}}= & \sqrt{\frac{715-\frac{(141)^{2}}{30}}{30-1}} \\
S_{O_{1}} & =\sqrt{1,80344} \\
S_{O_{1}} & =1,3429
\end{aligned}
$$

\section{2.- Construcción de la Distribución de frecuencias 03}

\begin{tabular}{cccccc}
\hline$O_{3_{i}}$ & $\mathrm{fi}$ & $\mathrm{hi}$ & $O_{3_{i}} * f i$ & $O_{3_{i}}^{2}$ & $O_{3_{i}}^{2} * f i$ \\
\hline 3 & 4 & 0,17 & 12 & 9 & 36 \\
4 & 10 & 0,43 & 40 & 16 & 160 \\
5 & 3 & 0,13 & 15 & 25 & 75 \\
6 & 2 & 0,09 & 12 & 36 & 72 \\
7 & 4 & 0,17 & 28 & 49 & 196 \\
TOTALES & 23 & 1,00 & 107 & 135 & 539 \\
\hline
\end{tabular}

Fuente: Secretaría del Centro de Idiomas ESPOCH

2. Cálculo de los Estadígrafos

- Media Aritmética (Promedio $\mathrm{O}_{3}$ )

$$
\begin{aligned}
& \overline{O_{3}}=\frac{\sum_{i=3}^{n} O_{i_{3}}}{n} \\
& \overline{O_{3}}=\frac{107}{23}
\end{aligned}
$$


- Desviación Estándar de $\mathrm{O}_{3}$

$$
\overline{O_{3}}=4,65217
$$

$$
\begin{gathered}
S_{O_{3}}=\sqrt{\frac{\sum f i * O_{3}^{2}-\frac{\left(\sum f i * O_{3}^{2}\right)^{2}}{n}}{n-1}} \\
S_{O_{3}}=\sqrt{\frac{539-\frac{107^{2}}{23}}{22}} \\
S_{O_{3}}=1,36876
\end{gathered}
$$

Del análisis del pre test a ambos grupos se obtiene la información que se presenta en la siguiente tabla.

\section{Resumen estadígrafos pre test}

\begin{tabular}{lcc}
\hline \multicolumn{1}{c}{ Pre Test } & $O_{1}$ & $O_{3}$ \\
\hline Estadígrafo & & \\
Promedio & 4,7 & 4,65217 \\
Desviación Estándar & 1,3429 & 1,3688 \\
No. de Estudiantes & 30 & 23 \\
\hline
\end{tabular}

Fuente: Secretaría del Centro de Idiomas ESPOCH

Para poder determinar si entre los dos grupos se pueden hallar diferencias significativas en el conocimiento de los temas pre vistos en los contenidos y conocimientos de los estudiantes previo al proceso experimental, se utilizó un estimador puntual éste permitió identificar un punto crítico para la diferencia de medias llamado cota de error.

1. Cálculo del estimador $\left(\overline{O_{1}}-\overline{O_{3}}\right)=0.04783$

La desviación estándar de medias es $\sigma\left(\overline{O_{1}}-\overline{O_{3}}\right)=\sqrt{\frac{\sigma_{\bar{O}_{1}}^{2}}{n \overline{O_{1}}}+\frac{\sigma_{\overline{O_{3}}}^{2}}{n_{\overline{O_{3}}}}}$

$$
\begin{aligned}
& \sigma\left(\overline{O_{1}}-\overline{O_{3}}\right)=\sqrt{\frac{1,3429^{2}}{30}+\frac{1,3688^{2}}{23}} \\
& \sigma\left(\overline{O_{1}}-\overline{O_{3}}\right)=\sqrt{\frac{1,80}{30}+\frac{1,87}{23}} \\
& \sigma\left(\overline{O_{1}}-\overline{O_{3}}\right)=0,38 \\
& \text { Siendo la cota de error } 2\left(\sigma\left(\overline{O_{2}}-\overline{O_{4}}\right)\right)=0,75
\end{aligned}
$$

En esta cota se determina la decisión en que "si la diferencia de medias es mayor que la cota de error, entonces hay diferencia entre los promedios de las muestras; caso contrario se afirmaría que no hay diferencia significativa en el promedio de las muestras", como se puede observar la cota de error 0.753 es mayor a la diferencia de medias 0.38 entonces, se puede afirmar que los estudiantes no tienen diferencias significativas en los promedios obtenidos en el pre test, con lo que se inicia el experimento en igualdad de condiciones. 
Análisis estadístico del post test para la verificación de hipótesis

1. Distribución de frecuencias de $\mathrm{O}_{2}$

\begin{tabular}{cccccc}
\hline$O_{2_{i}}$ & $\mathrm{fi}$ & $\mathrm{hi}$ & $O_{2_{i}} * f i$ & $O_{2_{i}}^{2}$ & $O_{2_{i}}^{2} * f i$ \\
\hline 4 & 6 & 0,20 & 24 & 16 & 96 \\
5 & 9 & 0,30 & 45 & 25 & 225 \\
6 & 9 & 0,30 & 54 & 36 & 324 \\
7 & 5 & 0,17 & 35 & 49 & 245 \\
8 & 1 & 0,03 & 8 & 64 & 64 \\
TOTALES & 30 & 1,00 & 166 & 190 & 954 \\
\hline
\end{tabular}

Fuente: Secretaría del Centro de Idiomas ESPOCH

1. Cálculo de estadígrafos

- Media Aritmética (Promedio $\mathrm{O}_{2}$ )

$$
\begin{aligned}
& \overline{O_{2}}=\frac{\sum_{i=1}^{n} O_{1_{i}} * f i}{n} \\
& \overline{O_{2}}=\frac{166}{30} \\
& \overline{O_{2}}=5,5333
\end{aligned}
$$

- Desviación Estándar

$$
\begin{aligned}
& S_{O_{2}}=\sqrt{\frac{\sum f i * O_{2_{i}}^{2}-\frac{\left(\sum f i * O_{2_{i}}\right)^{2}}{n-1}}{n}} \\
& S_{O_{2}}=\sqrt{\frac{954-\frac{(166)^{2}}{30}}{30-1}} \\
& S_{O_{2}}=1,1058
\end{aligned}
$$

- Varianza $\sigma_{\overline{O_{2}}}^{2}=1,22$

2. Distribución de frecuencias de $\mathrm{O}_{4}$

\begin{tabular}{cccccc}
\hline$O_{4_{i}}$ & $\mathrm{fi}$ & $\mathrm{hi}$ & $O_{4_{i}} * f i$ & $O_{4}^{2}$ & $O_{4_{i}}^{2} * f i$ \\
\hline 6 & 1 & 0,04 & 6 & 36 & 36 \\
7 & 1 & 0,04 & 7 & 49 & 49 \\
8 & 5 & 0,22 & 40 & 64 & 320 \\
9 & 12 & 0,52 & 108 & 81 & 972 \\
10 & 4 & 0,17 & 40 & 100 & 400 \\
TOTALES & 23 & 1,00 & 201 & 330 & 1777 \\
\hline
\end{tabular}

Fuente: Secretaría del Centro de Idiomas ESPOCH 
1. Cálculo de los Estadígrafos

- Media Aritmética (Promedio $\left.\mathrm{O}_{4}\right)$

$$
\begin{aligned}
& \overline{O_{4}}=\frac{\sum_{i=1}^{n} O_{4_{i}} * f i}{n} \\
& \overline{O_{4}}=\frac{201}{23} \\
& \overline{O_{4}}=8,7391
\end{aligned}
$$

- Desviación Estándar de $\mathrm{O}_{4}$

$$
\begin{aligned}
S_{O_{4}} & =\sqrt{\frac{\sum f i * O_{4_{i}}^{2}-\frac{\left(\sum f i * O_{4_{i}}\right)^{2}}{n-1}}{n}} \\
S_{O_{4}} & =\sqrt{\frac{1777-\frac{(201)^{2}}{23}}{23-1}} \\
S_{O_{4}} & =0,9637
\end{aligned}
$$

- Varianza

$$
\sigma_{\overline{O_{4}}}^{2}=0,93
$$

Del análisis del post test a los grupos de control y experimental se obtiene la información que se detalla en la siguiente tabla.

\section{3.- Resumen estadígrafos post test}

\begin{tabular}{lcc}
\hline \multicolumn{1}{c}{ Pre Test } & $O_{4}$ \\
\hline Estadígrafo & & \\
Promedio & 5,5333 & 8,7391 \\
Desviación Estándar & 1,1058 & 0,9637 \\
Varianza & 1,22 & 0,93 \\
No. de Estudiantes & 30 & 23 \\
\hline
\end{tabular}

Fuente: Secretaría del Centro de Idiomas ESPOCH

La desviación estándar de la diferencia de medias es $\left(\overline{O_{2}}-\overline{O_{4}}\right)=3,2$

Siendo la cota de error $2\left(\sigma\left(\overline{\mathrm{O}_{2}}-\overline{\mathrm{O}_{4}}\right)\right)=0,569$

En esta cota se determina la decisión en que "si la diferencia de medias es mayor que la cota de error, entonces hay diferencia entre los promedios de las muestras, caso contrario se afirmaría que no hay diferencia significativa en los promedios de las muestras" como se puede observar la cota de error 0.569 es menor a la diferencia de medias 3.2. Se puede afirmar que los estudiantes tienen diferencias significativas en los promedios del post test. 


\section{Prueba de hipótesis}

Tomamos las calificaciones del examen (post test) aplicado a dos grupos distintos a los que se les enseñó con metodologías diferentes: al Grupo de Control $(\mathrm{Gc})$ se le aplicó el método tradicional auxiliado del uso de los recursos tecnológicos de uso cotidiano y al Grupo Experimental (Ge) se le aplicó como metodología didáctica los CHUNKING FLASH CARDS.

1 Redacción de hipótesis

H1: Existe una diferencia significativa entre la media de las calificaciones del Grupo de Control (Gc) y la media de las calificaciones del Grupo Experimental (Ge ).

H0: NO existe una diferencia significativa entre la media de las calificaciones del Grupo de Control (Gc) y la media de las calificaciones del Grupo Experimental (Ge).

2 Determinar el nivel de significancia

Por tratarse de un problema de carácter social se elige un alfa igual al 5\% $\alpha=0.05$

Como se aplicará el nivel de significancia del 5\%, se trata de un ensayo a dos colas.

\section{Gráfico 5. Campana de Gauss}

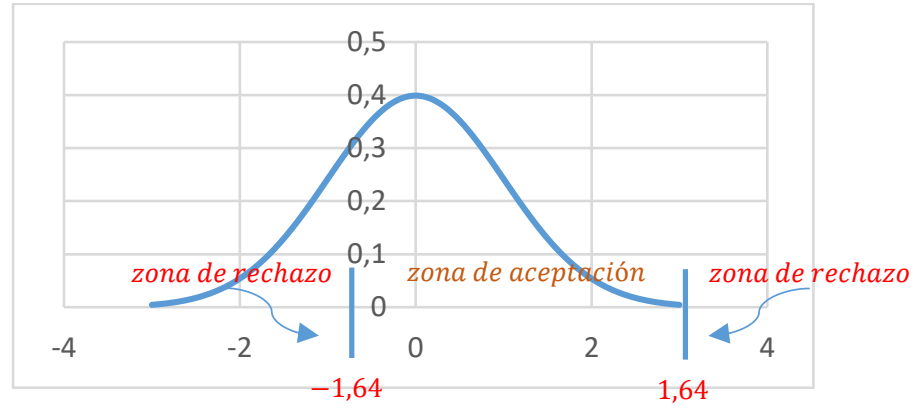

Fuente: Gran Enciclopedia Larousse 1987

"-1,64<= z <= 1,64"

3 Determinar la elección de la prueba estadística

Si vamos a evaluar a los dos grupos al mismo momento, se trata de un estudio de tipo transversal en el cual la variable aleatoria es una variable numérica que son las calificaciones obtenidas en el Post test o examen final de conocimientos; por tanto, la prueba para este caso es la prueba t-student para muestras independientes. 
ISSN: 2602-8085

\begin{tabular}{lrr}
\hline & Variable 1 & \multicolumn{1}{c}{ Variable 2 } \\
\hline Media & 5,533333333 & 8,7391304 \\
Varianza & 1,222988506 & 0,9288538 \\
Observaciones & 30 & 23 \\
Diferencia hipotética de las medias & 0 & \\
Grados de libertad & 50 & \\
Estadístico t & $-11,25352241$ & \\
$\mathrm{P}(\mathrm{T}<=\mathrm{t})$ una cola & $1,30373 \mathrm{E}-15$ & \\
Valor crítico de $t$ (una cola) & 1,675905025 & \\
$\mathrm{P}(\mathrm{T}<=\mathrm{t})$ dos colas & 0,0000000000000026 & \\
Valor crítico de $t$ (dos colas) & 2,008559112 & \\
\end{tabular}

4 Formular la regla de decisión

Aceptar H0: cuando t calculado pertenezca al intervalo

$$
-1,64<=\mathrm{z}<=1,64
$$

5 Tomar la decisión respecto a H0: e interpretar el resultado

La decisión es RECHAZAR la hipótesis nula y ACEPTAR la hipótesis alterna; pues el valor de $t$ calculado es 2,008559112 que se localiza fuera del intervalo $(-1.64,1.64)$ de $\mathrm{z}$ : "La aplicación de la estrategia Chunking Flashcards Si influye en el desarrollo de la competencia léxica en inglés en los estudiantes del Segundo Nivel paralelos " $\mathrm{H}$ " y "O" del Centro de Idiomas de la Escuela Superior Politécnica de Chimborazo en el período Abril-Agosto 2017".

Después de realizar el análisis estadístico de los resultados obtenidos por los estudiantes del Segundo Nivel de Inglés del Centro de Idiomas de Escuela Superior Politécnica de Chimborazo, se puede concluir que existe diferencias significativas entre los datos obtenidos para una enseñanza tradicional y una enseñanza con el uso de la estrategia Chunking Flashcards.

\section{Validación}

La validación se la realizó con el paralelo "G" del Segundo Nivel del Centro de Idiomas de la Escuela Superior Politécnica de Chimborazo. El instrumento usado para este evento fue Alfa de Cronbach, coeficiente que sirve para medir la fiabilidad de una escala de medida.

\begin{tabular}{ll}
\multicolumn{1}{l}{$\alpha=\frac{k}{k-1}\left[1-\frac{\sum S_{i}^{2}}{S_{T}^{2}}\right]$} & \\
& \\
K & 60 \\
SUM.VAR & 9.6172414 \\
V.T. & 73.389655 \\
SECCIÓN 1 & 1.0169492 \\
SECCIÓN 2 & 0.8689564 \\
ALFA DE CRONBACH & 0.8836845
\end{tabular}


El nivel de confianza obtenido 0.88368454 aproximado a una cifra decimal es 0.9 y como éste está por encima de 0.7 que es punto de referencia el instrumento de evaluación es una herramienta que ha sido aceptada por tener un nivel de confianza aceptado. El análisis de la información obtenida mediante el diseño cuasi experimental permitió realizar diversos análisis estadísticos como el análisis de varianza, la prueba " $\mathrm{t}$ " entre otras, así la prueba de la hipótesis general de investigación se realizó con "t-student" para valorar si hay diferencias significativas puesto que se trató de una hipótesis de diferencia entre grupos, cuya fórmula fue la siguiente:

$$
t=\frac{\bar{X}-\bar{Y}}{\sqrt{\frac{(n-1) S_{1}^{2}+(m-1) S_{2}^{2}}{n+m-2}} \sqrt{\frac{1}{n}+\frac{1}{m}}}
$$

Para poder concretar el análisis se partió de las calificaciones obtenidas en cada grupo, con esta información se elaboraron tablas estadísticas la primera para tabular en porcentajes los ítems respondidos según el grado de satisfacción de la respuesta, una segunda tabla que agrupa en porcentajes los puntajes obtenidos por destrezas y una tercera tabla resumen de calificaciones por destrezas evaluadas. Esto permitió realizar el análisis parcial de satisfacción en el pre y post test y la interpretación de resultados como producto de la construcción de tablas de frecuencia. Las destrezas analizadas en esta investigación fueron:

- $\quad$ Formación de palabras raíces

- Formación de palabras derivaciones

- $\quad$ Conjuntos de palabras partes del habla

- $\quad$ Conjuntos de palabras contenidos temáticos

- Asociación de palabras escritura

- $\quad$ Asociación de palabras significado

- Asociación de palabras aspecto socio-cultural

\section{Conclusiones}

- La estrategia Chunking Flashcards puede ser enseñada en cualquier nivel de estudio. Se dice que esta estrategia permite descomponer una palabra en otras derivadas facilitando la reproducción, el almacenamiento en la memoria y la evocación de vocabulario en situaciones de aprendizaje.

- Se concluye que, el nivel de la competencia léxica está determinado por el rango de las calificaciones obtenidas en el post test que elevó la medida de 5,5 a 8,7, porque los Chunking Flashcards como ayuda visual favorecen en los estudiantes a que el aprendizaje sea más significativo permitiéndoles la fijación permanente en la memoria y reduciendo el tiempo de respuesta sobre lo aprendido aspecto que se vio reflejado en las respuestas al cuestionario aplicado en el post test.

- El nivel de competencia está determinado por la diferencia de medias de las calificaciones entre el Grupo de Control (Gc) y la media de las calificaciones del Grupo Experimental (Ge). Al existir diferencias significativas entre los datos obtenidos de la enseñanza 
tradicional y la enseñanza utilizando la estrategia Chunking Flascards se puede concluir que el uso de los Chunking Flashcards como estrategia incide favorablemente en la adquisición de la competencia léxica con los estudiantes del Segundo Nivel de Idiomas de la Escuela Superior Politécnica de Chimborazo.

- También se concluye que, el Chunking reduce el tiempo dedicado al aprendizaje y amplia el vocabulario como parte de la competencia léxica, debido a que la estrategia Chunking Flashcards ayudó a organizar los elementos en grupos significativos, en donde en lugar de percibir letras aisladas, percibieron y recordaron su agrupación significativa ayudada a través de las Flashcards

\section{Referencias bibliográfica}

Apostilla. (2014). "Concepto definiciones . Educacion P -definista.

Biava, M. L., \& Segura, A.L. (25 de 02 de 2019). Por qué es importante saber el idioma. Obtenido de cepjuanxxiii.edu.ar: http://cepjuanxxiii.edu.ar

Cabanilla, G. (10 de 11 de 2018). extra.ec/opinion. Obtenido de extra.ec/opinion: https://www.extra.ec/opinion/dhakhdkjshakjdhasjlkdlsahldjkhslajdsakda-KE2464840

Callejas, M. M. (1986). La investigacion en la formacion del profesor universitario: entre la teoria yla practica. Colombia: Colociencias Vol. 20, No. 4, diciembre, pp 3-12.

Camarco, M., Clavo, G., Franco, M., Vergara, M., Londoño, S., Zapata, F., \& Garavito, C.,. (2018). las necesidades de formacion permantente del docente . Eduacion $y$ Educadores, volumen 7, 80 .

Castelli, E. (2016). Formacion de palabras: composicion, derivacion y parasitesis. Galakia.

Ceballos, K., Dàvila, N., Espioza, J. \& Ramìrez, M. (2014). Factores que inciden en el aprendizaje del idioma inglès en los alumnos de segundo año de lla ciduad de Chillan. Chillan: Univeersidad del Bio Bio.

Chomsky, N. (1965). Aspects of the Theory of Syntax. Cambridge.

Cohn, M. (2009). Helping Your Teen-age Student: What Parents Can Do to Improve Reading and Study Skills. Estados Unidos.

Colmenarez, J. (2015). Ingles corocito. Obtenido de Ingles corocito. http://inglescorocito.blogspot.com/2015/04/partes-del-habla.html.

Dansereaua. (1986). Que son las estrategias de aprendizaje. Cientifica Universitaria.

De Miguel García, M. L. (02 de 2004). La enseñanza del léxico del español como lengua extranjera. Resultados de una encuesta sobre la metodología aplicada en el aula. Obtenido de marcoele.com: http://marcoele.com/revista/index.php/marcoele/article/view/1/1 
Díaz, A. y Quiroz, R. . (1998). Enseñanza cognitiva y estratégica en ciencias naturales para el desarrollo de la habilidad de solución de problemas en contaminación ambiental. . Colombia: Revista Educacion y Pedagogia, 10 (21), 97-115.

ELE, R. D. (2007). marcoele.com. Obtenido de http://marcoele.com/revista/index.php/marcoele/article/view/1/1

F. Reimers y L. Tiburcio. (1996). Educación, ajuste y reconstrucción: opciones para el cambio. París,. Paris- Ginebra: UNESCO.

Fernandez, H. (1964). Didactica. España.

Garcia,Ros., R., Clemente, A., \& Pèrez, Gonzalez, F.. (1994). Intervencion escolar en estrategias de aprendizaje. Valencia: CSV.

Gómez Molina, J. (1997). “El léxico y su didáctica. Una propuesta metodológica” . en REALE, 7, pp. 69-94.

González, A., Pulido, M. y Díaz, A. . (2005). Modelo pedagógico de la Escuela de Idiomas. Medellin : Escuela de Idiomas, Editorial Universidad de Antioquia.

Gonzàlez, M. E. (1996). Construyendo un modelo pedagogico para la Universidad de Medellin . Medellin.

Higueras, M. (2008). "Nuevas tecnicas para ensenar lexico, $V$ Encuentro practico de Profesores de ELE Wurzburg. . Wurzburg.

Hymes, D. (1971). On Communicative Competence. Philadelphia: University of Pennsylvania Press.

Jaimechango. (24 de 02 de 2009). Obtenido de Importancia-del-ingles-en-la-educacion: Disponible en https://es.slideshare.net

Kahneman. (2013). Fundamentos Psicologicos dea Actividada Cardiovascular y oculomotora çen P.A.G Raul Cabestrero. Madrid: Editorial digital, pág. 285.

LaHora. (07 de 10 de 2008). lahora.com.ec. Obtenido de lahora.com.ec: https://lahora.com.ec/noticia/782474/importancia-del-idioma-ingls-en-la-actualidad-

Larousse, P. (1987). Gran Enciclopedia Larousse. Francia: Editorial Planeta, Barcelona.

Mercedes. (19 de 05 de 2010). Espanolenamerica.wordpress.com. Obtenido de https://espanolenamerica.wordpress.com/2010/05/19/competencia-lexica/

Ministerio de Educación, C. y. ( 2002). Marco común europeo de referencia para las lenguas: aprendizaje, enseñanza y evaluación, . Madrid: Anaya.

Ministerio de Educación, C. y. (2002). Marco común europeo de referencia para las lenguas: aprendizaje, enseñanza evaluación. . Madrid: : MCER/Anaya.

Miranda, J. (1994). La Formación de Palabras en Español. Salamaca. España: Ediciones Colegio de España. 
Oxford, R. . (1990). Language Learning Strategies. What every Teacher should Know. . Boston,: Heinle \& Heinle Publishers.

Oxford, R. y Cohen A., . (1992). Language learning strategies: Crucila issues of concepts and classification. Massachusetts:: Applied Language Learning, 7 (1-2), 25-45.

Pablo. (30 de 04 de 2007). La Guía. Lengua. . Obtenido de lengua.laguia2000: https://lengua.laguia2000.com

Pérez, J., \& Gardney, A. . (2009). Definición.De. . Obtenido de https://definicion.de.

Rodrìguez, Y. (23 de 09 de 2014). Enseñanza del inglès desde la pedagogia de las emociones. Obtenido de vinculando.org/psicologia psi: http://vinculando.org

UNIVERSIA. (02 de 08 de 2017). noticias.universia.es. Obtenido de noticias.universia.es: http://noticias.universia.es/cultura/noticia/2017/08/02/1154707/ingles-idiomauniversal.html

WoodwarSpanish. (15 de 09 de 2017). www.spanish. Obtenido de https://www.spanish.cl/vocabulario/familia-de-palabras.htm

Zabert, A. (2010). Estrategias de Aprendizaje. PIIS Escuela Leloir. 


\section{PARA CITAR EL ARTÍCULO INDEXADO.}

Barragán Murillo, R. de los Ángeles, Herrera Andrade, Z., Colcha Guashpa, E., Guano, D., \& Rodríguez Arellano, N. (2019). Desarrollo de la competencia léxica en el idioma inglés aplicando la estrategia chunking. Ciencia Digital, 3(2.6), 308-331. https://doi.org/10.33262/cienciadigital.v3i2.6.569

\section{Ciencia \\ LDigital}

El artículo que se publica es de exclusiva responsabilidad de los autores y no necesariamente reflejan el pensamiento de la Revista Ciencia Digital.

El artículo queda en propiedad de la revista y, por tanto, su publicación parcial y/o total en otro medio tiene que ser autorizado por el director de la Revista Ciencia Digital.
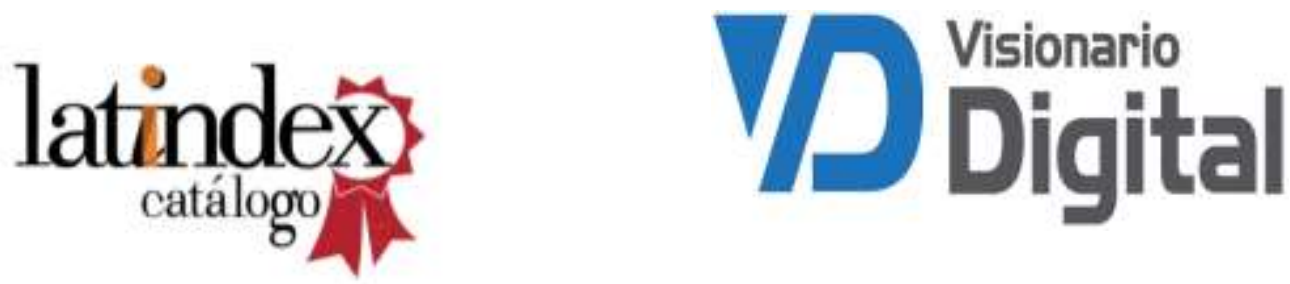